\title{
Dehydration of Benzyl Alcohols in Phosphonium Ionic Liquids: Synthesis of Ethers and Alkenes
}

\author{
Hassan A. Kalviri ${ }^{\mathrm{a}}$ and Francesca M. Kerton ${ }^{\mathrm{a}}{ }^{\mathrm{*}}$ \\ a Centre for Green Chemistry and Catalysis, Department of Chemistry, Memorial University of Newfoundland, St. \\ John’s, Newfoundland, Canada A1B 3X7. E-mail:fkerton@mun.ca; Fax:+1-709-8643702; Tel: +1-709-8648089
}

Received: 30 ${ }^{\text {th }}$ May 2011 Accepted: $8^{\text {th }}$ August 2011

Supporting information for this article is available on the WWW under http://dx.doi.org/10.1002/adsc.20110445.

Abstract. Dehydration of benzylic alcohols has been studied in several phosphonium ionic liquids in the absence of any metal catalysts. Benzyl ethers and alkenes were obtained from primary and secondary benzylic alcohols in good to excellent yields for these reactions. Commercially available hydrophobic phosphonium ionic liquids containing the trihexyl(tetradecyl)phosphonium cation paired with six different anions were used for the reactions under microwave irradiation. The interaction of the substrate with the ionic liquid was investigated using different NMR techniques, such as NOESY NMR. The effects of cation and anions on the behaviour of these ionic liquids in the reactions were studied in order to understand the mechanism. A catalytic cycle is proposed involving activation of the benzyl alcohol by the phosphonium cation.

Keywords: dehydration; benzyl ethers; alkenes; phosphonium ionic liquids; NOESY NMR; catalyst

\section{Introduction}

Imidazolium ionic liquids have been widely studied and used in many types of reactions. ${ }^{[1]}$ In some cases, imidazolium ionic liquids have acted as both catalyst and solvent. ${ }^{[2-4]}$ Previously, we reported dehydrative etherifications of benzylic alcohols in imidazolium ionic liquids, specifically, [BMIm] $\mathrm{PF}_{6}$ (1-butyl-3methylimidazolium hexafluorophosphate) in the presence of Pd catalysts. ${ }^{[5]}$ We chose to study such reactions in ionic liquids because these solvents display an exceptional ability to stabilize ionic intermediates. Phosphonium ionic liquids have been studied to a lesser extent although they possess some advantages over imidazolium ionic liquids. Phosphonium ionic liquids can be prepared without halide contamination, which is an important factor for the halogen sensitive reagents. ${ }^{[6,7]}$ Furthermore, phosphonium ionic liquids are more thermally stable than imidazolium and quaternary ammonium salts. ${ }^{[8,}$ 9] Also, tetraalkylphosphonium salts are much less acidic than imidazolium ionic liquids. ${ }^{[10,}$ 11] Additionally, imidazolium ionic liquids are not completely inert in some reactions (reagents can potentially interact with the pi-system and/or the acidic hydrogens on the ring periphery). ${ }^{[12]}$ However, it has also recently been reported that phosphonium ionic liquids can participate in reactions. ${ }^{[13-15]} \mathrm{We}$ have seen this in our own research where Pd(II) salts can be reduced to yield $\operatorname{Pd}(0)$ nanocrystals in phosphonium ionic liquids without adding any reducing agents. ${ }^{[13]}$ In this case, the phosphonium cation acts as the reducing agent.
Many phosphonium ionic liquids are commercially available and have been successfully used as reaction media ${ }^{[8,10,16]}$ and catalysts ${ }^{[17-19]}$ in a variety of organic syntheses. They have also found industrial uses. ${ }^{[20,21]}$ To the best of our knowledge, reports concerning the use of phosphonium ionic liquids as catalysts contain limited details about the mechanism of catalysis. For the oxychlorination reaction, ${ }^{[18]}$ Perosa et al. proposed that the nitrate anion is acting as the catalyst.

Dehydration reactions are becoming increasingly important in order to reduce the oxygen content of biomass feedstocks and convert them into more valuable materials for chemical and allied industries. Low selectivity and use of harsh reaction conditions are the main problems associated with dehydration reactions of alcohols. For example, sulfuric acid and $p$-toluenesulfonic acid are among the strong acids that are used for these reactions, ${ }^{[22 a, 23]}$ which need a neutralization process at the end of the pipeline. Heterogeneous acid catalysts have also been used with some success. ${ }^{[24,25]}$ Furthermore, using alcohols as feedstocks for the preparation of ethers is desirable, as waste production (mainly alkali metal salts) and use of toxic alkylating agents are two hazards encountered in classical ether syntheses. ${ }^{[22 b]}$

Through our previous studies, ${ }^{[5]}$ we became interested in using phosphonium ionic liquids as reaction media in the dehydration of benzylic alcohols to yield benzyl ethers and alkenes. Unlike our previous studies using imidazolium ionic liquids, we have since found that there is no need for Pd catalysts in phosphonium ionic liquids for these 
dehydration reactions and the phosphonium cation can catalyze these reactions.

Herein, dehydration reactions of benzylic alcohols are reported that can be performed within a short reaction time via microwave irradiation. The reaction conditions are very simple and highly atom economic, since there is no need for metal catalysts and alkylating agents and the ionic liquids are recyclable. Also a catalytic cycle for the role of phosphonium ionic liquids in these dehydration reactions will be presented.

\section{Results and Discussion}

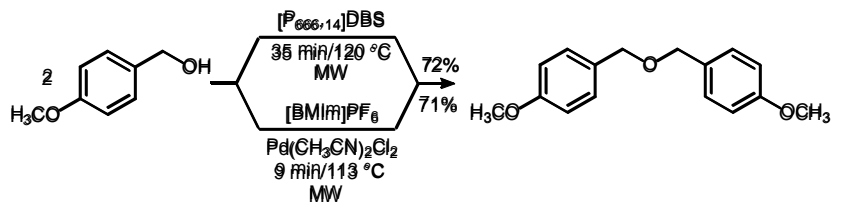

Scheme 1. Dehydrative etherification reaction of 4methoxybenzyl alcohol in $\left[\mathrm{P}_{666,14}\right] \mathrm{DBS}$ and $\left[\mathrm{BMIm}^{\mathrm{P}} \mathrm{PF}_{6}\right.$.

In our previous studies, very low conversions were observed in the absence of palladium for dehydrative etherification reactions in imidazolium ionic liquids, ${ }^{[5]}$ however, we have since discovered that the reaction can be performed without any metal catalyst in phosphonium ionic liquids (Scheme 1). Furthermore, $\left[\mathrm{BMIm} \mathrm{PF}_{6}\right.$ was not completely stable under the reaction conditions, as HF was released via hydrolysis of the ionic liquid and our initial studies showed greater stability for phosphonium ionic liquids. As a starting point, 4-methoxybenzyl alcohol was used as the substrate. Several phosphonium ionic liquids containing $\left[\mathrm{P}_{666,14}\right]^{+}$, trihexyl(tetradecyl)phosphonium cation, and six different counter ions were used as the reaction media (for their complete names and more information see Table 1). Among these phosphonium ionic liquids, $\left[\mathrm{P}_{666,14}\right]$ DBS was revealed as having the greatest compatibility with the reactions studied in terms of higher yields and ease of product isolation.

Figure 1 shows the progress of dehydrative etherification of 4-methoxybenzyl alcohol in $\left[\mathrm{P}_{666,14}\right] \mathrm{DBS}$ in the presence and absence of $\mathrm{Pd}\left(\mathrm{CH}_{3} \mathrm{CN}\right)_{2} \mathrm{Cl}_{2} \quad(5 \mathrm{~mol} \%$ with respect to 4methoxybenzyl alcohol). For this experiment, separate reaction mixtures were prepared with an alcohol to ionic liquid mole ratio of $0.65( \pm 0.05)$. The reaction mixtures were heated under microwave conditions at $120{ }^{\circ} \mathrm{C}$ for different reaction times as indicated in Figure 1. Higher yields were observed at shorter reaction times in the presence of palladium, however in the absence of palladium higher yields could be reached at longer reaction times. In the presence of palladium at prolonged reaction times product decomposition occurs ${ }^{[5]}$ and benzyl chloride is formed presumably from the chloride present in the palladium precursor. Without palladium, the reaction progress can be seen to plateau. We attribute this to the increasing water content of the reaction mixture. Under the reaction conditions studied no considerable improvement in yields was observed in the presence of water scavengers, such as molecular sieves, and anhydrous sodium sulfate. Kappe et al. have previously noted that molecular sieves are not efficient water scavengers under microwave assisted reaction conditions. ${ }^{[26]}$

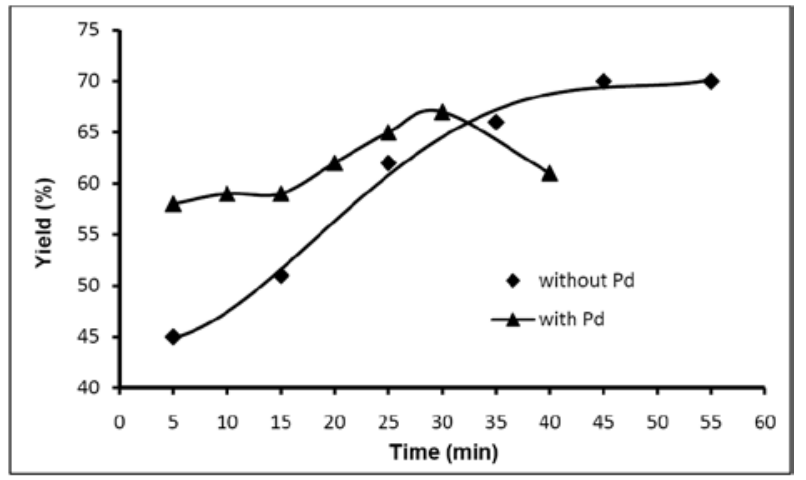

Figure 1. Reaction progress for dehydrative etherification of 4-methoxybenzyl alcohol at $120{ }^{\circ} \mathrm{C}$ in $\left[\mathrm{P}_{666,14}\right] \mathrm{DBS}$ in the presence of $5 \mathrm{~mol} \% \operatorname{Pd}\left(\mathrm{CH}_{3} \mathrm{CN}\right)_{2} \mathrm{Cl}_{2}$ and with no palladium.

In the aforementioned experiments, the concentration of 4-methoxybenzyl alcohol was determined based on ${ }^{1} \mathrm{H}$-NMR spectra of the reaction mixtures. For these experiments, a plot of $\ln [4-$ methoxybenzyl alcohol] vs. time gives a straight line (Figure 2). Therefore, the ionic liquid catalyzed dehydrative etherification reaction under these conditions is first order with respect to benzyl alcohol.

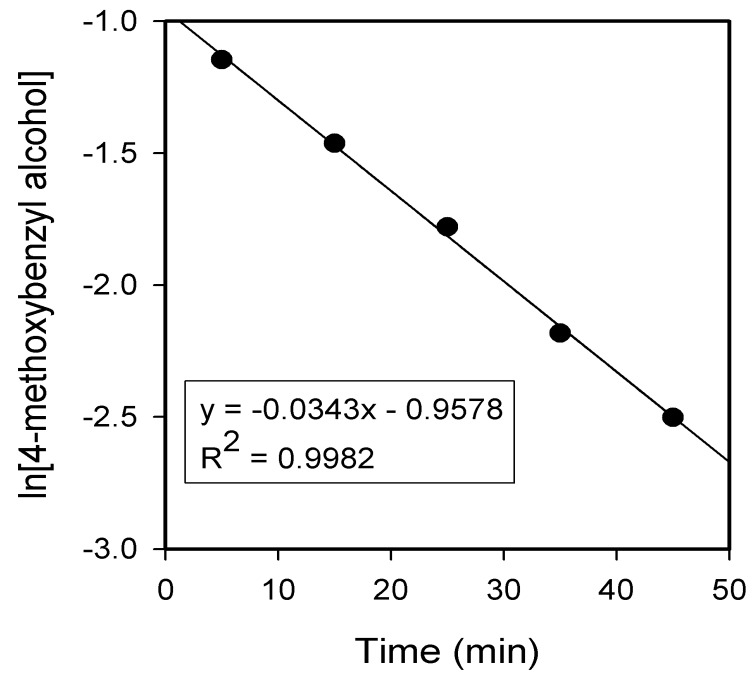

Figure 2. Plot of $\ln [4-m e t h o x y b e n z y l$ alcohol] vs. time for dehydrative etherification in $\left[\mathrm{P}_{666,14}\right] \mathrm{DBS}$ at $120{ }^{\circ} \mathrm{C}$. 
Table 1. Names and some general physicochemical properties of the trihexyl(tetradecyl)phosphonium ionic liquids that were used in this work. Data were collected from the cited references.

\begin{tabular}{|c|c|c|c|}
\hline Ionic liquid & Anion name & $\begin{array}{c}\text { Physical state at room } \\
\text { temperature }\end{array}$ & $\begin{array}{c}\text { Approximate therma } \\
\text { stability }\left({ }^{\circ} \mathrm{C}\right)\end{array}$ \\
\hline$\left[\mathrm{P}_{666,14}\right] \mathrm{Cl}$ & Chloride & Liquid, $T_{\mathrm{g}}=-56^{\circ} \mathrm{C}^{[11]}$ & $335^{[27]}$ \\
\hline$\left[\mathrm{P}_{666,14}\right] \mathrm{Br}$ & Bromide & Liquid $^{[16 c]}$ & $320^{[16 c]}$ \\
\hline$\left[\mathrm{P}_{666,14}\right]\left[\mathrm{NTf}_{2}\right]$ & Bis(trifluoromethylsufonyl)amide & Liquid, $T_{\mathrm{g}}=-76^{\circ} \mathrm{C}^{[11]}$ & $380^{[27]}$ \\
\hline$\left[\mathrm{P}_{666,14}\right] \mathrm{DBS}$ & Dodecylbenzene sulfonate & Liquid $^{\left[16 c^{2}\right.}$ & $350^{[28]}$ \\
\hline$\left[\mathrm{P}_{666,14}\right]\left[\mathrm{N}(\mathrm{CN})_{2}\right]$ & Dicyanamide & Liquid, $T_{\mathrm{g}}=-67^{\circ} \mathrm{C}^{[11]}$ & $360^{[27]}$ \\
\hline$\left[\mathrm{P}_{666,14}\right]\left[\left(\mathrm{i}-\mathrm{C}_{8}\right)_{2} \mathrm{PO}_{2}\right]$ & Bis(2,4,4-trimethylphenyl)phosphinate & Liquid $^{[16 c]}$ & $340^{[16 c]}$ \\
\hline
\end{tabular}

The etherification reaction was also performed on reaction mixtures with different concentrations of 4methoxybenzyl alcohol. These results were in agreement with the first order nature of the reaction (Table S1 in Supporting Information). Table S1 shows that higher yields were obtained at higher alcohol concentrations.

Several substrates were screened for this reaction and the results are presented in Table 2. Table 2 shows that higher yields were obtained for electron rich substrates (entries 1-5). No conversion was observed for control reactions, neat alcohol and no ionic liquid, under these conditions. Since the reactions were performed under batch conditions in closed vessels, the released water accumulated in the system and based on the Le Châtelier's principle the reaction was prohibited from achieving completion. No etherification was observed for aliphatic alcohols or phenols. However, asymmetric etherification was possible between phenols and benzyl alcohols but with low yields and poor selectivities. For example, $16 \%$ yield was obtained for the phenylbenzylether from condensation of 4-methoxybenzyl alcohol and 4-methoxyphenol at $150{ }^{\circ} \mathrm{C}$ (for GC-MS analysis see Figure S1)

Table 2. Etherification reaction of different benzylic alcohol substrates in $\left[\mathrm{P}_{666,14}\right] \mathrm{DBS}$ under microwave irradiation.
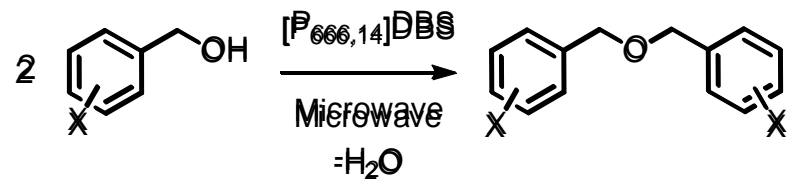

\begin{tabular}{clcc}
\hline Entry & \multicolumn{1}{c}{$\mathrm{X}$} & Yield $^{\text {a) }}[\%]$ & Time $[\min ] /$ Temp $\left[{ }^{\circ} \mathrm{C}\right]$ \\
\hline 1 & $4-\mathrm{MeO}$ & 72 & $35 / 120$ \\
2 & $3-\mathrm{MeO}$ & 53 & $25 / 200$ \\
3 & $4-\mathrm{EtO}$ & 50 & $20 / 140$ \\
4 & $4-\mathrm{Me}$ & 61 & $20 / 180$ \\
5 & $2-\mathrm{Me}$ & 63 & $20 / 180$ \\
6 & $\mathrm{H}$ & 34 & $60 / 180$ \\
7 & $4-\mathrm{Cl}$ & 26 & $45 / 170$ \\
\hline
\end{tabular}

a) Yields were calculated using ${ }^{1} \mathrm{H}-\mathrm{NMR}$ spectroscopy with acetophenone or mesitylene as standards.

Alkenes were produced as the major products for dehydration reactions of secondary benzylic alcohols which have $\beta$-hydrogens (Table 3). Different catalytic systems have been previously reported for this transformation. For example, recently $\mathrm{Re}_{2} \mathrm{O}_{7}$ or iodine under solvent-free conditions were reported as efficient catalysts for the dehydration of benzylic alcohols. $^{[29,30]}$ Also, aromatic alkenes can have biological properties. For example, some of the products in this paper (methoxy arylalkenes) are building blocks of asarones, which have bactericide and pesticide activities and can be used to treat diphtheria and typhoid. ${ }^{[31]}$ Since the alkene bond in indene is more stable than that in 4-methoxy styrene and styrene, (internal double bonds which are more substituted are more stable than terminal double bonds ${ }^{[22 c]}$ higher yields were observed for the dehydration of 1-indanol than 1-(4methoxyphenyl)ethanol and 1-phenylethanol. The lower yield in entry 5 compared to entry 6 is, possibly, because of polymerisation of styrene in $\left[\mathrm{P}_{666,14}\right] \mathrm{DBS}$. After reaction, most mixtures were transparent dark yellow-orange solutions while for entry 5 the reaction mixture was opaque. No attempt was made to fully characterize the insoluble by-product. The product from the reaction mixture in Table 3 , entry 2 was vacuum distilled $\left(3 \mathrm{~h}, 70\right.$ mtorr and $\left.110{ }^{\circ} \mathrm{C}\right)$ and the IL was reloaded with (1) and 99\% yield was obtained (Table 3, entry 3). For a picture of the vacuum distillation apparatus see Figure S2. This recycled ionic liquid was also used for dehydrative etherification of 4-methoxybenzyl alcohol and 71\% yield was obtained, which is comparable with the reaction in fresh $\left[\mathrm{P}_{666,14}\right] \mathrm{DBS}$ (Table 2, entry 1 ). It is interesting that the ionic liquid can be re-used in a different dehydration to that which for it was originally used in without any problems.

\section{Effect of pH}

Dehydrative etherification of 4-methoxybenzyl alcohol was also performed in five other phosphonium ionic liquids containing the same cation as $\left[\mathrm{P}_{666,14}\right]$ DBS but differing anions. The $\mathrm{pH}$ of these ionic liquids was measured to probe the effect of acidity on the reaction mechanism. The results have been summarized in Figure 3. Figure 3 shows that all these phosphonium ionic liquids have acidic properties and cover roughly three different acidic $\mathrm{pH}$ ranges. Although the acidic properties of these ionic liquids can come from possible impurities, ${ }^{[21]}$ it has been shown that these ionic liquids have an inherent 
acidic nature. ${ }^{[15,32]}{ }^{1} \mathrm{H}$ and ${ }^{31} \mathrm{P}$ NMR spectra and elemental analysis did not indicate the presence of any impurities in these ionic liquids. Surprisingly, high and low yields were observed for different ionic liquids across the $\mathrm{pH}$ range 1.5-4.5. These results highlight the point that there is no correlation between the $\mathrm{pH}$ of the ionic liquids and the reaction yield. Therefore, the reactions are not acid catalyzed. The method that was used for the $\mathrm{pH}$ measurements did not directly measure the acidity of the ionic liquids (see the experimental section for more details). ${ }^{[32]}$ However, it provides a reasonable estimate for the relative acidities of the ionic liquids. Also, no correlation between the $\mathrm{pH}$ of the ionic liquids and yield was seen in the dehydration of secondary benzylic alcohols (Table 3). Significantly lower yields were obtained in $\left[\mathrm{P}_{666,14}\right] \mathrm{Cl}$ compared with the other two ionic liquids studied.

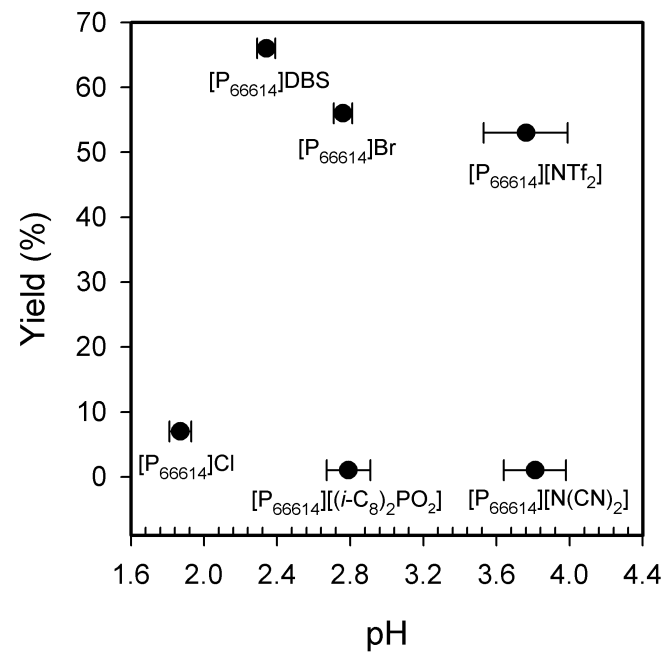

Figure 3. Percent yield for dehydrative etherification reaction of 4-methoxybenzyl alcohol in six different phosphonium ionic liquids. Horizontal axis shows the measured $\mathrm{pH}$ for the corresponding neat ionic liquids. $\left[\mathrm{P}_{666,14}\right]$ : trihexyl(tetradecyl)phosphonium; DBS: Dodecylbenzene sulfonate; $\mathrm{Br}$ : Bromide; [NTf ${ }_{2}$ ]: Bis(trifluoromethylsulfonyl)amide; Cl: Chloride; $\left[\left(i-\mathrm{C}_{8}\right)_{2} \mathrm{PO}_{2}\right]: \quad \operatorname{Bis}(2,4,4$-trimethylpentyl)phosphinate; $\left[\mathrm{N}(\mathrm{CN})_{2}\right]$ : Dicyanamide

\section{Chloride Ion Levels}

Seddon et al. have reported the importance of chloride impurities on the physical properties of ionic liquids. ${ }^{[33]}$ We wondered if the low yield obtained using $\left[\mathrm{P}_{666,14}\right] \mathrm{Cl}$ was due to chloride ions and if the other two ionic liquids $\left(\left[\mathrm{P}_{666,14}\right]\left[\left(i-\mathrm{C}_{8}\right)_{2} \mathrm{PO}_{2}\right]\right.$ and $\left.\left[\mathrm{P}_{666,14}\right]\left[\mathrm{N}(\mathrm{CN})_{2}\right]\right)$ which gave low yields were contaminated with chloride ions. Therefore, chloride measurements were performed on the six phosphonium ionic liquids studied. All contained less than 0.3 wt\% chloride, except $\left[\mathrm{P}_{666,14}\right] \mathrm{Cl}$ which contained $8.02 \mathrm{wt} \%$ chloride in agreement with its formulation.

\section{Effect of Water}

All six phosphonium ionic liquids studied are hydrophobic, however their water capacities are not the same. Water solubility in some of the ionic liquids is increased due to the formation of reverse micelles. For example, a high water capacity, higher than $14 \mathrm{wt} \%$, was reported for $\left[\mathrm{P}_{666,14}\right]\left[\left(i-\mathrm{C}_{8}\right)_{2} \mathrm{PO}_{2}\right]$ and $\left[\mathrm{P}_{666,14}\right] \mathrm{Cl}$, while for $\left[\mathrm{P}_{666,14}\right] \mathrm{Br}, 4.5 \mathrm{wt} \%$ was reported. ${ }^{116,}{ }^{34]}$ However, we did not measure the water content of the ionic liquids in our study. This factor could have a dramatic impact on the efficiency of the ionic liquids in dehydration reactions. In these reactions water is produced and the more hydrophobic the ionic liquid is, the higher yield should be obtained. In order to confirm the negative effects of water, the dehydrative etherification of 4methoxybenzyl alcohol in $\left[\mathrm{P}_{666,14}\right] \mathrm{DBS}$ was performed in the presence of intentionally added 20 wt\% water. The reaction was carried out under microwave conditions $\left(15 \mathrm{~min}\right.$ at $\left.120{ }^{\circ} \mathrm{C}\right)$ with an alcohol to IL mole ratio of 0.7 . 24\% yield was obtained for this reaction while, under the same reaction conditions, $64 \%$ yield was achieved for the reaction without added water. Therefore, the use of hydrophobic ionic liquids is a driving force for enhancing the progress of the reactions towards the products.

\section{Effect of Ionicity}

Another possibility is that the ionic properties of the ionic liquids could be a driving force for these reactions via their ability to stabilize ionic intermediates. Some of the ionic liquids, for example $\left[\mathrm{P}_{666,14}\right] \mathrm{Cl}$, have more molecular-related properties and can have a more closely packed network extended structure with the cations and anions interacting closely. On the other hand, some other anions, like $\left[\mathrm{NTf}_{2}\right]$ are less coordinating and can lead to more ionic properties within the ionic liquids, i.e. the cation and anion are less closely associated with one another within the liquid phase. ${ }^{[35]}$ Therefore, $\left[\mathrm{P}_{666,14}\right]\left[\mathrm{NTf}_{2}\right]$ should be more suitable for reactions with polar intermediates than the chloride ionic liquid, as it will be better able to stabilize the ionic intermediates. The less ionic nature of the chloride ionic liquid was confirmed when a reaction mixture of $\left[\mathrm{P}_{666,14}\right] \mathrm{Cl}$ was vacuum distilled at 80 mtorr (3 hours at $80{ }^{\circ} \mathrm{C}$ ). The presence of this ionic liquid in the gas phase demonstrates that $\left[\mathrm{P}_{666,14}\right] \mathrm{Cl}$ can behave in a molecular fashion, where there is significant bonding between the chloride and phosphonium ions. ${ }^{1} \mathrm{H}$ - and ${ }^{31} \mathrm{P}$-NMR spectra (Figure S3) of the distilled material showed the presence of $\left[\mathrm{P}_{666,14}\right] \mathrm{Cl}$ in addition to the desired product. However, under the same vacuum distillation conditions no peaks related to the ionic liquid were observed for samples prepared in $\left[\mathrm{P}_{666,14}\right]\left[\mathrm{NTf}_{2}\right]$ or $\left[\mathrm{P}_{666,14}\right] \mathrm{DBS}$. Thus, these two ionic liquids are less volatile and more ionic in nature compared with $\left[\mathrm{P}_{666,14}\right] \mathrm{Cl}$. Therefore, they can provide more suitable environments for ionic reaction intermediates and yields are thereby enhanced. 
Table 3. Dehydration reactions of secondary benzylic alcohols that have $\beta$-hydrogens.

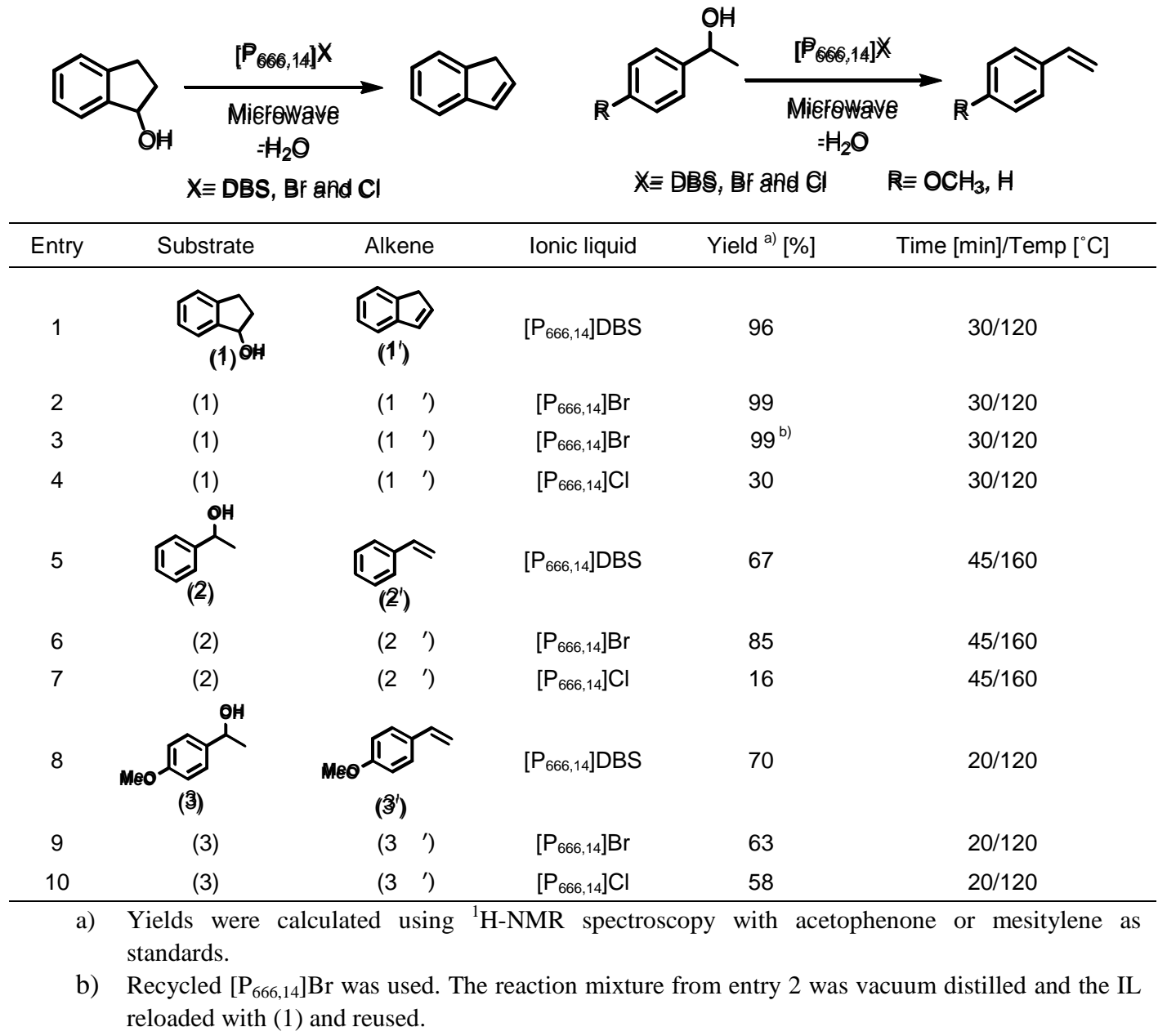

\section{Interactions of Substrate with Phosphonium Cation}

NMR studies were performed on samples of the reaction mixtures and neat ionic liquids in order to collect more data to use in proposing a reaction mechanism. ${ }^{1} \mathrm{H}-\mathrm{NMR}$ spectra of the phosphonium ionic liquids were obtained with higher resolution when using deuterated acetone rather than $\mathrm{CDCl}_{3}$ as the solvent (Figures S4 and S5). This demonstrates the proton exchange ability of all the methylene groups on the alkyl chains within the ionic liquids. The acidic nature of the protons on the methylene groups adjacent to the phosphorus atom has been reported previously, ${ }^{[15]}$ but significant levels of exchange are also observed for protons farther from the phosphorus atom. In order to investigate the interactions of alcohols with phosphonium ionic liquids, NOESY (Nuclear Overhauser Effect Spectroscopy) NMR was used (Figure 4). The integrations in the NOESY spectrum showed the greatest interactions of the $\mathrm{CH}_{2}$ benzylic protons with the methylene groups $d, \quad b$ and $c$ and fewer interactions with the methylenes adjacent to the phosphorus $a$ (Figure 4). Regarding the choice of ionic liquid used in this study, of the ionic liquids used by us and that afforded good yields (Tables 2 and 3), $\left[\mathrm{P}_{666,14}\right]\left[\mathrm{NTf}_{2}\right]$ has the lowest viscosity. Therefore, better molecular tumbling in this ionic liquid provides lower signal broadening and higher resolution NMR spectra, especially for the neat sample used in the NOESY NMR experiment. Furthermore, $\left[\mathrm{P}_{666,14}\right]\left[\mathrm{NTf}_{2}\right]$ does not have alkyl chains within its anion, which simplifies its NMR spectra compared with $\left[\mathrm{P}_{666,14}\right] \mathrm{DBS}$.

${ }^{1} \mathrm{H}-\mathrm{NMR}$ spectra of neat $\left[\mathrm{P}_{666,14}\right]\left[\mathrm{NTf}_{2}\right]$ with different concentrations of 4-methoxybenzyl alcohol were in agreement with the results from NOESY NMR (Figure S6). The resolution of the ${ }^{1} \mathrm{H}-\mathrm{NMR}$ spectra decreased at higher alcohol concentration for the methylene groups farther from the phosphorus (Figure S6, spectrum 3), while for the methylene groups adjacent to the phosphorus, better resolution was obtained at higher alcohol concentrations. This means that in these systems, there are more interactions and exchange between the alcohol and the methylenes labelled $b, c$ and $d$ (Figures 4 and S4) than methylenes labelled $a$. 


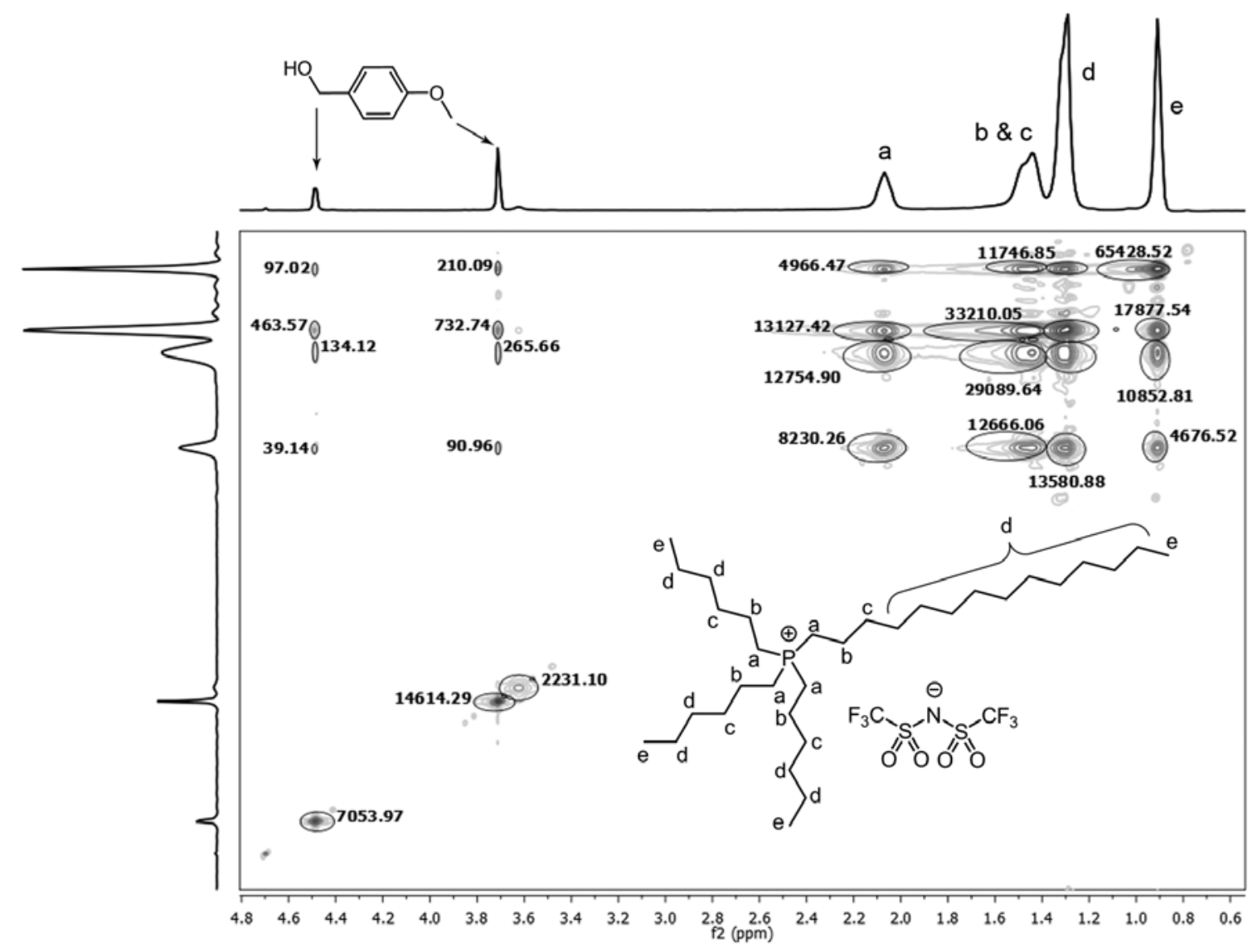

Figure 4. NOESY spectrum (selected region) of 4-methoxybenzyl alcohol in $\left[\mathrm{P}_{666,14}\right]\left[\mathrm{NTf}_{2}\right]$. $\mathrm{D}_{2} \mathrm{O}$ was used as NMR lock solvent in a coaxial NMR tube. Numbers on the contours in the figure indicate integrals for the cross peak resonances.

All the aforementioned discussion has been summarised in a catalytic cycle that is proposed in Figure 5. Further support for the presence of ionic intermediates can be obtained by considering the resolution and chemical shifts of the protons in ${ }^{1} \mathrm{H}$ NMR spectra of the ionic liquids in the presence and absence of 4-methoxybenzyl alcohol (Figure S6). A significant shift $(0.14 \mathrm{ppm})$ to lower frequency was observed in the presence of alcohol for the protons adjacent to the phosphorus (protons labelled $a$ ) than the other protons $(0.08 \mathrm{ppm}$ for protons labelled $b$ and c). This demonstrates an increase in the partial electron density next to the above-mentioned protons with increasing alcohol concentration. This would be expected for ionic intermediates such as those proposed in Figure 5.

A similar mechanism could be followed by secondary alcohols with $\beta$-hydrogens. However, as intramolecular elimination is kinetically favoured for these molecules over intermolecular substitution, these reactions yield alkenes rather than ethers.

A deuterium isotope exchange experiment was also performed on a sample of $\left[\mathrm{P}_{666,14}\right]\left[\mathrm{NTf}_{2}\right]$, the same ionic liquid that was used for the NOESY NMR experiment. The procedure was modified from the method that was used by Chu et al. for $\left[\mathrm{P}_{666,14}\right] \mathrm{Cl}^{[15]}$ The ionic liquid, $16.6 \mathrm{mg}$, was dissolved in $600 \mu \mathrm{L}$
$\mathrm{CD}_{3} \mathrm{OD}$ and $\mathrm{D}_{2} \mathrm{O}$ (1:1 volume ratio) and a ${ }^{1} \mathrm{H}$ NMR spectrum obtained. A solution of $\mathrm{NaOD}(0.4 \mathrm{M}, 50$ $\mu \mathrm{L}$ ) was added to the abovementioned solution and after $40 \mathrm{~min}$ a second spectrum obtained. The integral ratios for the different methylene groups were normalized relative to the terminal methyl hydrogens. The results showed that protons of the three methylene groups adjacent to the phosphorus, $\mathrm{H}_{\mathrm{a}}, \mathrm{H}_{\mathrm{b}}$ and $\mathrm{H}_{\mathrm{c}}$ in Figure 4, in the ionic liquid had undergone deuterium exchange (their integrals decreased over time). $10 \%$ of " $\mathrm{H}$ " had undergone exchange for each of these but was negligible for all other $\mathrm{H}$ environments.

The stability of phosphonium cations in phosphonium ionic liquids toward bases strongly depends on the size and strength of the base. ${ }^{[36,37]}$ In the case of larger bases or reagents, the inner protons of the cation, $\mathrm{H}_{\mathrm{a}}$, are less accessible and the more accessible protons, $\mathrm{H}_{c}$, etc. have significant interactions with these reagents. Therefore, in the case of benzylic alcohols and the mechanism proposed here it is plausible that because of steric hindrance, the inner protons, $\mathrm{H}_{\mathrm{a}}$, have lower interactions with the benzylic protons. The proposed mechanism matches with the NMR evidence, but more experimental and possibly theoretical evidence is needed to exclude the possibility of an ylide-catalyzed reaction, where $\mathrm{H}_{\mathrm{a}}$ is 
abstracted by the alcohol. Ylides have been shown to act as reactive intermediates in a variety of organic reactions and they can also activate alcohols. ${ }^{[38]}$ At this time we have obtained no evidence (e.g. ${ }^{31} \mathrm{P}$ NMR data) to support ylide formation, but it cannot be excluded. It is likely that any reactive intermediates, e.g. an ylide, would be present on a very short time scale before reacting and therefore would not be observable by NMR spectroscopy.

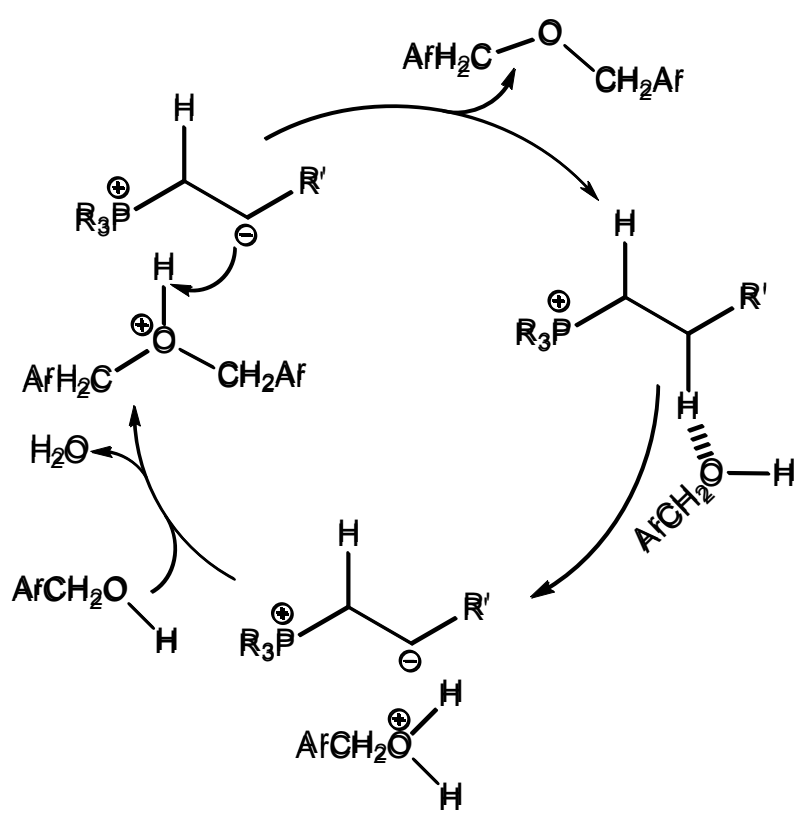

Figure 5. Proposed catalytic cycle for dehydrative etherification of benzylic alcohols in phosphonium ionic liquids. The counterion of the ionic liquid has been omitted for clarity. $\mathrm{H}^{+}$abstraction may also be occurring at the $\mathrm{C} \alpha$ to the $\mathrm{P}$ atom.

\section{Conclusions}

A simple and atom economic method for the conversion of benzylic alcohols to their corresponding ethers and alkenes has been developed. The method is compatible with the principles of green chemistry, since alkylating agents and metal catalysts are avoided. Phosphonium ionic liquids, which are more thermally stable and occasionally cheaper than imidazolium ionic liquids have been used as both the reaction media and catalysts. The ionic liquids are recyclable through vacuum distillation of the reaction mixtures. Both solvent screening and NMR studies support the formation of an ionic intermediate. The protons in $\beta, \gamma$ and $\delta$ positions of the alkyl chains in the ionic liquids are seen via NOESY NMR to undergo ready exchange with the acidic protons of the alcohol. In the presence of $\mathrm{NaOD}, \mathrm{CD}_{3} \mathrm{OD}$ (a smaller alcohol) and $\mathrm{D}_{2} \mathrm{O}, \alpha, \beta$ and $\gamma$ protons undergo deuterium exchange. Theses results show that phosphonium ionic liquids can act as the catalyst and reaction media in dehydration reactions. Further studies are needed in order to fully understand the mechanism and in order to scale up the reactions and increase substrate scope. One possible route for increasing yields and improving scale-up potential would be to use a supported version of these ionic liquids and perform dehydration reactions in a continuous-flow manner.

\section{Experimental Section}

\section{General Information and Instrumentation}

The ionic liquids $\left[\mathrm{P}_{666,14}\right]$ DBS (Cyphos phosphonium IL 202) and $\left[\mathrm{P}_{666,14}\right] \mathrm{Cl}$ (Cyphos phosphonium IL 101) were gift samples from Cytec Industries and the other ionic liquids were purchased from Sigma-Aldrich. Ionic liquids were purified following the procedure of Walsby, Clyburne and co-workers. ${ }^{14]}$ Alcohols were purchased from Alfa Aesar and used as received. A Biotage Initiator 2.5 microwave reactor was employed for the reactions. 0.5-2 $\mathrm{mL}$ microwave vials with PTFE seals were used for all the experiments. A Bruker AVANCE $500 \mathrm{MHz}$ and a Bruker AVANCE III $300 \mathrm{MHz}$ spectrometer equipped with XBB probe were used for the NMR experiments. ${ }^{1} \mathrm{H}-\mathrm{NMR}$ spectra were referenced to tetramethylsilane as an internal standard and ${ }^{31} \mathrm{P}-\mathrm{NMR}$ spectrum was referenced to external $81 \% \mathrm{H}_{3} \mathrm{PO}_{4}$. GC-MS spectra were recorded on an Agilent 7890A GC system coupled with an Agilent 5975C MS detector that was equipped with a capillary column DB-5 (column length: $30.0 \mathrm{~m}$ and column diameter: $0.25 \mathrm{~mm}$ ). Elemental analysis and chloride ion measurements of the ionic liquids were performed by Canadian Microanalytical Service Ltd. (Delta, BC, Canada).

\section{Experimental for Dehydrative Etherification Reaction}

In a typical reaction, $\left[\mathrm{P}_{666,14}\right] \mathrm{DBS}(0.5070 \mathrm{~g})$ was degassed under vacuum. 4-Methoxybenzyl alcohol (0.0979 g) was added to the ionic liquid in a 0.5-2.0 mL Biotage microwave vials and capped under nitrogen flow. The solution was stirred for a few minutes before putting in the microwave reactor. The reaction mixture was heated for 35 min at $120{ }^{\circ} \mathrm{C}$. The absorptivity on the MW was set at "high" for all the samples and the microwave power reached 43-45 W (in this case) during the reaction. No colour change was observed in the reaction mixture upon heating, however droplets of water could be observed near the top of the microwave vial after reactions. Yields were calculated based on ${ }^{1} \mathrm{H}$-NMR spectra using mesitylene or acetophenone as standards. ${ }^{1} \mathrm{H}-\mathrm{NMR}$ chemical shifts related to $\mathrm{CH}_{2}$ benzylic (in benzyl ethers) and alkene bonds (in alkenes) have been presented in Tables S2 and S3 and the data are comparable with reported values. ${ }^{[5,39]}$ In the case of styrene and 4-methoxystyrene, the products were compared with commercially available standards. The progress of the reactions was monitored using thin layer chromatography (TLC). GC-MS analysis could be performed on the TLC spots extracted with diethyl ether or direct extraction of the reaction mixture, if possible, to identify the products formed (e.g. Figures S7 to S12). Product separation can be performed either by solvent extraction or distillation. Solvent extraction is possible for $\left[\mathrm{P}_{666,14}\right] \mathrm{Cl}$ with $3: 1$ volume ratio of heptane-water. No degradation was observed for the ionic liquids during the reactions studied. ${ }^{1} \mathrm{H}$ NMR spectra of the ionic liquids, before and after the reactions, did not show any changes in the relative intensities and chemical shifts of the resonances assignable to the ionic liquids. Also high resolution mass 
spectrometry on $\left[\mathrm{P}_{666,14}\right]$ DBS did not show any differences between the mass spectra of the ionic liquid before and after the reaction. The same procedure can be used for the dehydration reactions of alcohols $1-3$ (Table 3 ) to yield alkenes. Yields were calculated relative to the alcohols using ${ }^{1} \mathrm{H}-\mathrm{NMR}$ spectroscopy with acetophenone (for $\left[\mathrm{P}_{666,14}\right] \mathrm{DBS}$ ) or mesitylene (for $\left[\mathrm{P}_{666,14}\right] \mathrm{Br}$ and $\left[\mathrm{P}_{666,14}\right] \mathrm{Cl}$ ) as the internal standards.

\section{General Procedure for $\mathrm{pH}$ Measurements of the Ionic Liquids}

A procedure similar to that reported by Deng et al. was used to measure the $\mathrm{pH}$ of the ionic liquids. ${ }^{[32]}$ In each case, $5.00 \mathrm{~mL}$ deionised water was added to $0.5 \mathrm{mmol}$ ionic liquid and the mixture was vigorously stirred for 2 minutes. The mixtures were passed through silica plugs and the aqueous phases were titrated with sodium hydroxide titrant. Each sample was titrated three times.

\section{Supporting Information}

Additional ${ }^{1} \mathrm{H}$ and ${ }^{31} \mathrm{P}-\mathrm{NMR}$ spectra, table $\mathrm{S} 1$, a photo of the vacuum distillation apparatus and some examples of GC-MS spectra are available in supporting information.

\section{Acknowledgements}

We acknowledge support from NSERC and Canada Foundation for Innovation in the form of a Discovery Grant, a Research Tools and Instrument Grant and a Leaders Opportunity Fund Award (F. $M$. K.). We also thank Memorial University for funding and Cytec Industries Inc. for samples of $\left[P_{666,14}\right] D B S$ and $\left[P_{666,14}\right] C l$. Dr. Céline Schneider (C-CART) is thanked for assistance with some NMR experiments.

\section{References}

[1] J. P. Hallett, T. Welton, Chem. Rev. 2011, 111, 35083576.

[2] T. Welton, Coord. Chem. Rev. 2004, 248, 2459-2477.

[3] S. Chowdhury, R. S. Mohan, J. L. Scott, Tetrahedron 2007, 63, 2363-2389.

[4] R. Sheldon, Chem. Commun. 2001, 2399-2407.

[5] H. A. Kalviri, C. F. Petten, F. M. Kerton, Chem. Commun. 2009, 5171-5173.

[6] L. G. Bonnet, B. M. Kariuki, Eur. J. Inorg. Chem. 2006, 437-446.

[7] B. Kirchner, Editor, Ionic Liquids in: Top. Curr. Chem. 2009, 290, 345.

[8] E. Janus, W. Stefaniak, Catal. Lett. 2008, 124, 105-110.

[9] K. Tsunashima, M. Sugiya, Electrochem. Commun. 2007, 9, 2353-2358.

[10] B. D. Powell, G. L. Powell, P. C. Reeves, Lett. Org. Chem. 2005, 2, 550-553.

[11] R. E. Del Sesto, C. Corley, A. Robertson, J. S. Wilkes, J. Organomet. Chem. 2005, 690, 2536-2542.
[12] A. Sharma, R. Kumar, N. Sharma, V. Kumar, A. K. Sinha, Adv. Synth. Catal. 2008, 350, 2910-2920.

[13] H. A. Kalviri, F. M. Kerton, Green Chem. 2011, 13, 681-686.

[14] T. Ramnial, S. A. Taylor, M. L. Bender, B. Gorodetsky, P. T. K. Lee, D. A. Dickie, B. M. McCollum, C. C. Pye, C. J. Walsby, J. A. C. Clyburne, J. Org. Chem. 2008, 73, 801-812.

[15] M. Tseng, H. Kan, Y. Chu, Tetrahedron Lett. 2007, 48, 9085-9089.

[16] a) T. Itoh, K. Kude, S. Hayase, M. Kawatsura, Tetrahedron Lett. 2007, 48, 7774-7777; b) V. I. Parvulescu, C. Hardacre, Chem. Rev. 2007, 107, 26152665; c) K. J. Fraser, D. R. MacFarlane, Aust. J. Chem. 2009, 62, 309-321; d) L. Ford, F. Atefi, R. D. Singer, P. J. Scammells, Eur. J. Org. Chem. 2011, 942-950; e) J. McNulty, J. J. Nair, A. Robertson, Org. Lett. 2007, 9, 4575-4578; f) J. McNulty, S. Cheekoor, T. P. Bender, J. A. Coggan, Eur. J. Org. Chem. 2007, 1423-1428; g) J. McNulty, J. J. Nair, S. Cheekoori, V. Larichev, A. Capretta, A. J. Robertson, Chem. Eur. J. 2006, 12, 9314-9322; h) J. McNulty, S. Cheekoori, J. J. Nair, V. Larichev, A. Capretta, A. J. Robertson, Tetrahedron Lett. 2005, 46, 3641-3644; i) D. A. Gerritsma, A. Robertson, J. McNulty, A. Capretta, Tetrahedron Lett. 2004, 45, 7629-7631; j) H. Cao, H. Alper, Org. Lett. 2010, 12, 4126-4129; k) Q. Yang, H. Alper, J. Org. Chem. 2010, 75, 948-950; l) H. Cao, L. McNamee, H. Alper, Org. Lett. 2008, 10, 5281-5284; m) Q. Yang, A. Robertson, H. Alper, Org. Lett. 2008, 10, 5079-5082; n) H. Cao, L. McNamee, H. Alper, J. Org. Chem. 2008, 73, 3530-3534.

[17] S. R. Sarda, W. N. Jadhav, S. U. Tekale, G. V. Jadhav, B. R. Patil, G. S. Suryawanshi, R. P. Pawar, Lett. Org. Chem. 2009, 6, 481-484.

[18] M. Noe, A. Perosa, M. Selva, L. Zambelli, Green Chem. 2010, 12, 1654-1660.

[19] S. R. Sarda, W. N Jadhav, A. S. Shete, K. B. Dhopte, S. M. Sadawarte, P. J. Gadge, R. P. Pawar, Synth. Commun. 2010, 40, 2178-2184.

[20] N. V. Plechkova, K. R. Seddon, Chem. Soc. Rev. 2008, 37, 123-150.

[21] C. J. Bradaric, A. Downard, C. Kennedy, A. J. Robertson, Y. Zhou, Green Chem. 2003, 5, 143-152.

[22] M. B. Smith, J. March, Ed.; In March's Advanced Organic Chemistry; John Wiley \& Sons: Hoboken, New Jersey, 2007, pp. a) 1513-1516 b) 529-532 c) 1497-1501.

[23] E. C. Wijnbelt, E. H. Hekelaar, Patent WO 2010110841, 2010.

[24] L. R. Pizzio, P. G. Vazquez, C. V. Caceres, M. N. Blanco, E. N. Alesso, M. I. Erlich, R. Torviso, L. Finkielsztein, B. Lantano, G. Y. Moltrasio, J. M. Aguirre, Catal. Lett. 2004, 93, 67-73. 
[25] A. Tarlani, A. Riahi, M. Abedini, M. M. Amini, J. Muzart, J. Mol. Catal. A: Chem. 2006, 260, 187-189.

[26] M. Baghbanzadeh, C. O. Kappe, Aust. J. Chem. 2009, 62, 244-249.

[27] Z. S. Breitbach, D. W. Armstrong, Anal. Bioanal. Chem. 2008, 390, 1605-1617.

[28] L. Yu, D. Garcia, R. Ren, X. Zeng, Chem. Commun. 2005, 2277-2279.

[29] T. J. Korstanje, J. T. B. H. Jastrzebski, R. J. M. Klein Gebbink, ChemSusChem 2010, 3, 695-697.

[30] G. Stavber, M. Zupan, S. Stavber, Tetrahedron Lett. 2006, 47, 8463-8466.

[31] R. Kumar, A. Sharma, N. Sharma, V. Kumar, A. K. Sinha, Eur. J. Org. Chem. 2008, 5577-5582.

[32] X. Cui, S. Zhang, F. Shi, Q. Zhang, X. Ma, L. Lu, Y. Deng, ChemSusChem 2010, 3, 1043-1047.

[33] K. R. Seddon, A. Stark, M. Torres, Pure Appl. Chem. 2000, 72, 2275-2287.

[34] J. Martak, S. Schlosser, Sep. Purif. Technol. 2007, 57, 483-494.
[35] a) M. J. Earle, J. M. S. S. Esperanca, M. A. Gilea, J. N. Canongia Lopes, L. P. N. Rebelo, J. W. Magee, K. R. Seddon, J. A. Widegren, Nature 2006, 439, 831-834; b) K. Ueno, H. Tokuda, M. Watanabe, Phys. Chem. Chem. Phys. 2010, 12, 1649-1658; c) V. Ermolaev, V. Miluykov, I. Rizvanov, D. Krivolapov, E. Zvereva, S. Katsyuba, O. Sinyashin, R. Schmutzler, Dalton Trans. 2010, 39, 5564-5571.

[36] T. Raminal, D. D. Ino, J. A. C. Clyburne, Chem. Commun. 2005, 325-327.

[37] S. Sowmiah, V. Srinivasadesikan, M. C. Tseng, Y. H. Chu, Molecules 2009, 14, 3780-3813.

[38] For example: L. Kürti, B. Czakó, In Strategic Applications of Named Reactions in Organic Synthesis, Background and Detailed Mechanisms; Elsevier Academic Press, California, 2005, pp. 16, 27, 104.

[39] J. S. Yadav, B. V. Subba Reddy, K. Shiva Shankar, T. Swamy, Tetrahedron Lett. 2009, 51, 46-48. 


\section{FULL PAPER}

Dehydration of Benzyl Alcohols in Phosphonium

Ionic Liquids: Synthesis of Ethers and Alkenes

Adv. Synth. Catal. Year, Volume, Page - Page

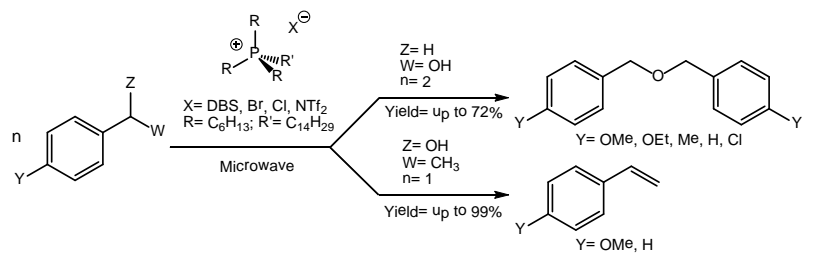

Hassan A. Kalviri and Francesca M. Kerton* 